\title{
Malignant eccrine spiradenoma with carcinomatous and sarcomatous elements
}

\author{
W G McCluggage, L J Fon, D O’Rourke, M Ismail, C M Hill, T G Parks, D C Allen
}

\begin{abstract}
A case of a 60 year old man with malignant eccrine spiradenoma involving the perineum is described. Areas of typical eccrine spiradenoma were admixed with carcinomatous and sarcomatous elements. Immunohistochemical and ultrastructural analysis revealed no evidence of epithelial differentiation in the sarcomatous areas. The tumour qualified for the designation carcinosarcoma arising in eccrine spiradenoma. The clinical course was aggressive with rapid development of nodal and pulmonary metastases.

(F Clin Pathol 1997;50:871-873)
\end{abstract}

Keywords: eccrine spiradenoma; carcinosarcoma

Eccrine spiradenoma is a generally benign skin appendage neoplasm that recapitulates primitive eccrine dermal ductular and secretory epithelium. Malignancy developing in an eccrine spiradenoma is a rare but well documented occurrence and in most cases is caused by carcinomatous transformation. ${ }^{12}$ Occasional cases of carcinosarcoma arising in eccrine spiradenoma have been described. ${ }^{3}$ We report a further case, including an ultrastructural and detailed immunohistochemical analysis.

Department of Pathology, Royal Group of Hospitals Trust, Belfast, UK W G McCluggage D O'Rourke C M Hill

Paediatric Surgery L J Fon

Department of Surgery, Downe Hospital, Downpatrick, UK M Ismail

Department of Surgery, Belfast City Hospital Trust, Belfast, UK

T G Parks

Department of Pathology

D C Allen

Correspondence to: Dr McCluggage, Department of Pathology, Royal Group of Hospitals Trust, Grosvenor Road, Belfast BT12 6BL, UK.

Accepted for publication 15 July 1997

\section{Case report}

A 60 year old man presented with a mass involving the skin of the left side of the perineum. The lesion had been present for approximately 10 years but had increased in size over the previous few months. Several smaller satellite nodules were present in the skin of the scrotum. An enlarged lymph node, $3 \mathrm{~cm}$ in diameter, was palpable in the left groin at the time of presentation. Ultrasound, computed tomography (CT), and magnetic resonance imaging of the abdomen and perineum revealed no evidence of metastatic disease. Biopsy was followed by resection that consisted of wide excision of the perineal mass and of the satellite nodules within the scrotal skin. Subsequently, block dissection of the left inguinal lymph nodes was performed. CT of the chest three months later revealed multiple pulmonary metastases.

\section{PATHOLOGICAL FINDINGS}

The surgical specimen consisted of an ellipse of skin measuring $15 \times 14 \mathrm{~cm}$ with $9 \mathrm{~cm}$ of subcutaneous tissue. Most of the specimen was involved by a necrotic tumour that extended deeply into the subcutaneous tissue. Several small satellite nodules were present in the dermis adjacent to the main lesion.
Histology showed an intact epidermis with no evidence of dysplasia. A tumour was present within the dermis and subcutaneous fat. This had a variegated appearance and included areas of typical eccrine spiradenoma (fig 1A). On high power examination two cell types were identified. Cells with round to ovoid nuclei containing evenly dispersed pale chromatin were admixed with smaller cells that had round hyperchromatic nuclei. Ductular structures were also present. In these areas, there was little nuclear pleomorphism and only an occasional mitotic figure was identified. Adjacent to the typical eccrine spiradenoma, carcinomatous areas were present (fig 1B). Here, groups and cords of epithelioid cells were surrounded by a fibrovascular stroma. Again two cell types and glandular structures were present. However, tumour cell nuclei were pleomorphic with prominent nucleoli and mitotic figures were easily identified (mitotic count in the region of 10 in 10 high power fields).

At the deep aspect of the neoplasm, sarcomatous areas were present (fig 1C). Tumour cells were spindle shaped with no glandular formation or other discernible arrangement. There was marked nuclear pleomorphism with many multinucleate tumour giant cells. Tumour cells contained moderate to abundant eosinophilic cytoplasm, but cross striations were not identified. Numerous mitotic figures were present (mitotic count in the region of 20 in 10 high power fields), and there were large areas of necrosis. No chondroid areas or areas of osteoid formation were identified.

Histology of several of the satellite lesions showed them to be composed of typical eccrine spiradenoma, located in the dermis.

Sectioning of the left inguinal lymph node dissection specimen revealed several nodes, one of which was enlarged and necrotic. Histology of this node showed it to be almost totally replaced by metastatic tumour that was extensively necrotic. In areas, the tumour had a carcinomatous appearance, similar to the carcinomatous areas within the skin neoplasm. However, elsewhere the tumour had a sarcomatous appearance, identical to the corresponding areas within the cutaneous neoplasm. The other nodes showed no evidence of metastatic tumour.

IMMUNOHISTOCHEMICAL FINDINGS

Tumour cells in areas of typical eccrine spiradenoma and in carcinomatous areas were diffusely positive for CAM5.2 (Becton Dickinson, Sunnyvale, California, USA) and EMA 


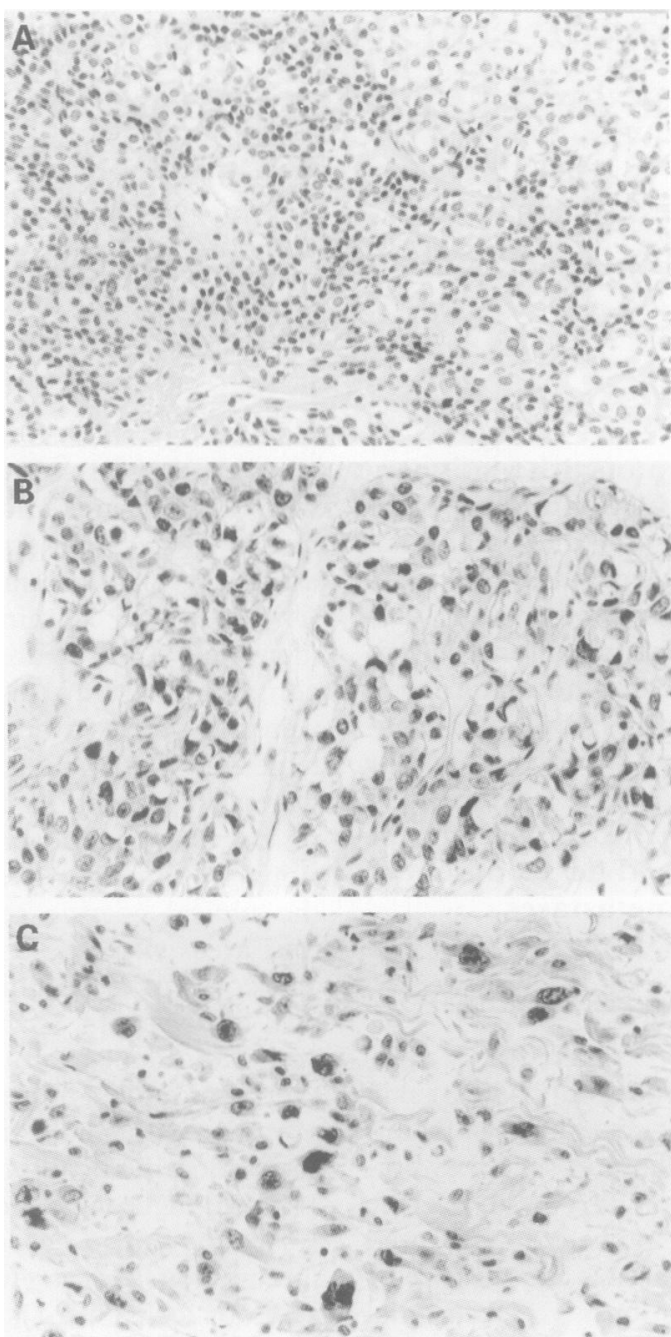

Figure 1 (A) Area of typical eccrine spiradenoma. (B) Carcinomatous area with epithelioid tumour cells exhibiting nuclear pleomorphism. (C) Sarcomatous area composed of pleomorphic spindle shaped cells with multinucleate tumour giant cells.

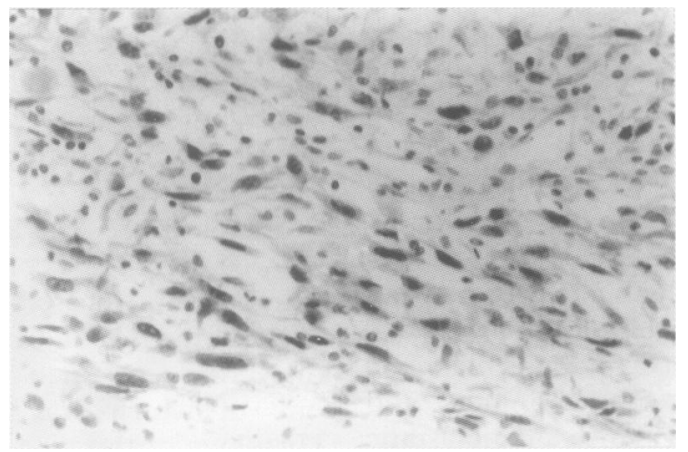

Figure 2 Large numbers of tumour cells in sarcomatous area exhibiting positive staining with $D O-7$.

(Dako, Copenhagen, Denmark), but negative for vimentin (Dako), desmin (Dako), and chromogranin A (Dako). There was weak positive staining of ductular structures for CEA (Dako). Significant numbers of tumour cells in these areas were positive for $S-100$ protein (Diagnostic Products Ltd, Abingdon, UK) and $\alpha$ smooth muscle actin (Sigma, Poole, UK). In sarcomatous areas, tumour cells were positive for vimentin but negative for CAM5.2, EMA, CEA, S-100 protein, desmin, $\alpha$ smooth muscle actin, and chromogranin A. In all three morphological areas, large numbers of tumour cell nuclei were positive with DO-7 (Novocastra, Newcastleupon-Tyne, UK) (fig 2).

ELECTRON MICROSCOPY

Ultrastructural examination of tissue that had been paraffin wax embedded showed cell preservation to be poor. In areas of typical eccrine spiradenoma and in carcinomatous areas groups of cells were surrounded by abundant basement membrane-like material. Adjacent cells were joined by well formed adhesion specialisations. The cytoplasm contained a range of organelles, and intracytoplasmic lumina lined by short microvillous projections were a prominent feature. Aggregated groups of intermediate filaments were present within the cytoplasm of several cells.

In the sarcomatous areas, spindle shaped cells, without intercellular relations, lay within a collagenous stroma. The cell cytoplasm contained a range of organelles, including abundant rough endoplasmic reticulum. There was no evidence of muscle or neural differentiation. The ultrastructural features were in keeping with non-specific fibroblast-like cells.

\section{Discussion}

Malignant neoplasms of sweat gland origin are rare. They may arise de novo or, more commonly, develop from a pre-existent and recognisably benign sweat gland neoplasm. The latter is the usual mode of development of malignant eccrine spiradenoma. In cases of malignant transformation, the typical clinical history, as in the present instance, is of a cutaneous lesion that has been present for several years, which undergoes some spontaneous alteration resulting in the patient seeking medical advice. Most cases of malignancy developing in eccrine spiradenoma have been carcinomas, ${ }^{12}$ although occasional cases of carcinosarcoma have been reported. ${ }^{3}$ McKee et al described two cases of carcinosarcoma arising from benign eccrine spiradenomas in middle aged women. ${ }^{3}$ In a search of the literature, we did not identify any other reports of this association. In both cases, areas of typical eccrine spiradenoma merged with a malignant neoplasm containing both epithelial and mesenchymal components. The sarcomatous component in one case showed osteocartilaginous and rhabdomyoblastic differentiation, and in the other only osteosarcoma was evident. In the present case, the malignant mesenchymal component comprised undifferentiated sarcoma with no discernible differentiation identified by immunohistochemical or ultrastructural analysis.

Mutation of the p53 tumour suppressor gene, located on the short arm of chromosome 17 , is among the most commonly detected genetic abnormalities in human neoplasia. Wild-type p53 in normal tissues is generally not detected by immunohistochemistry due to a combination of low expression and short half-life. Mutation of the p53 gene results in a conformational change in the protein that becomes stabilised, thus allowing for immunohistochemical detection. Overexpression of 
the $\mathrm{p} 53$ protein, as detected by immunohistochemistry, has been demonstrated by Biernat et al in the carcinomatous component of three cases of malignant eccrine spiradenoma. ${ }^{4}$ The authors found no positive staining in cases of typical benign eccrine spiradenoma or in the benign areas of the malignant tumours. Interestingly, in the present case, there was definite positive nuclear staining with DO-7 in the morphologically benign component as well as in the carcinomatous and sarcomatous areas. Biernat et al considered that accumulation of p53 protein may play an important role in the progression from benign to malignant eccrine spiradenoma, a hypothesis that would be supported by the immunohistochemical findings in the present case.

The neoplasm we describe contained recognisable epithelial and mesenchymal elements, both of which were malignant and thus qualified for the designation carcinosarcoma. Carcinosarcoma has occasionally been described as a primary tumour within the skin, without a pre-existing benign sweat gland neoplasm. ${ }^{5}$ Most reported cases have consisted of areas of basal cell carcinoma (with or without squamous elements) admixed with sarcomatous elements. It is probable that the mesenchymal component in cases of carcinosarcoma represents sarcomatous metaplasia in malignant epithelial cells. Ultrastructural examination in the present case did not reveal any evidence of epithelial differentiation in the sarcomatous areas. A prominent finding was the presence of intracytoplasmic lumina in the typical and carcinomatous areas. Intracytoplasmic lumina have been identified in other ultrastructural studies of eccrine spiradenoma. ${ }^{6}$

It is difficult to predict the prognosis of carcinosarcoma developing in eccrine spiradenoma as so few cases have been reported. Of the cases described by McKee et al, ${ }^{3}$ one patient died with pelvic and hepatic metastases within two years of diagnosis. The second patient remained alive and well 10 years following diagnosis and local resection. The rapid development of nodal and pulmonary metastases in the present case suggests an aggressive behaviour. It is of interest in the present case that the morphology of the nodal metastases mirrored that of the primary tumour with recognisable carcinomatous and sarcomatous components.

1 Evans HL, Su D, Smith L, Winkelmann RK. Carcinoma arising in eccrine spiradenoma. Cancer 1979;43:1881-4.

2 Wick MR, Swanson PE, Kaye VN, Pittelkow MR. Sweat gland carcinoma ex eccrine spiradenoma. Am $\mathcal{F}$ Dermato-

3 McKee PH, Fletcher CDM, Stavrinos P, Pambakian H. Carcinosarcoma arising in eccrine spiradenoma. A clinicopathologic and immunohistochemical study of two cases. Am f Dermatopathol 1990;12:335-43.

4 Biernat W, Kordek R, Wozniak L. Over-expression of p53 protein as an indicator of the malignant transformation in spiradenoma. Histopathology 1995;26:439-43.

5 Leen EJ, Saunders MP, Vollum DI, Keen CE. Carcinosarcoma of skin. Histopathology 1995;26:367-71.

6 Jitsukawa K, Sueki H, Sato S, Anzai T. Eccrine spiradenoma. An electron microscopic study. Am $\mathcal{f}$ Dermatopathol 1987;9:99-108.
Department of

Pathology, Kawasaki

Medical School,

Kurashiki, Japan

M Shimizu

T Manabe

$Y$ Monobe

M Hirokawa

T Moriya

Department of

Medicine (Division of

Gastroenterology)

T Matsumoto

$M$ Iida

Correspondence to: Dr Shimizu, Department of Pathology, Kawasaki Medical School, 577 Matsushima, Kurashiki City, Okayama, 701-01, Japan.

Accepted for publication 15 July 1997

\title{
$\beta_{2}$ Microglobulin haemodialysis related amyloidosis: distinctive gross features of gastrointestinal involvement
}

\author{
M Shimizu, T Manabe, T Matsumoto, Y Monobe, M Hirokawa, T Moriya, M Iida
}

\begin{abstract}
Two cases of $\beta_{2}$ microglobulin amyloidosis following long term haemodialysis found during necropsy are reported. The patients were 59 and 65 year old Japanese men, respectively. In both cases, systemic distribution of $\beta_{2}$ microglobulin amyloid deposits was observed. The gastrointestinal tract including the stomach, small intestine, and colon showed the distinctive gross feature of rippled appearance, which was characterised by serosal wrinkles along the muscle layer arrangement. These areas were confirmed to contain deposits of $\beta_{2}$ microglobulin in the muscularis propria. Although the outline of the muscle layers was preserved, most muscle fibres, encircled by the amyloid deposits,
\end{abstract}

were atrophic or had disappeared microscopically. In neither case could a definite diagnosis of amyloidosis be made while the patient was alive. Interestingly, the oesophagus presented less involvement compared to the remainder of the gastrointestinal tract. In comparison with the AA or AL type of amyloidosis, $\beta_{2}$ microglobulin haemodialysis related amyloidosis showed a rippled appearance of the serosal rather than mucosal changes, which may explain the difficulty in diagnosing amyloid deposits using biopsies of the gastrointestinal tract. (F Clin Pathol 1997;50:873-875)

Keywords: $\beta_{2}$ microglobulin; amyloidosis; haemodialysis; gastrointestinal tract; stomach; small intestine; colon 